\title{
The Physiological Value of Synthetic Fats
}

\section{By H. Kraut, Max Planck-Institut für Arbeitsphysiologie, Dortmund}

Fat is the first foodstuff which has been industrially produced by synthesis.* Pioneer experiments in the last century and after the first world war, carried through by British and German scientists, first showed the methods of obtaining long-chain fatty acids by air-oxidation of petroleum. It was found that catalytic oxidation was the most efficient and economical method. So far, the results of these investigations have not been used industrially. Fat shortage, which was the driving force behind such investigations, decreased quickly after the first world war. The interest in obtaining synthetic fats was revived in Germany about ro years later, and coincided with the development of a new starting material, the paraffin fraction of the Fischer-Tropsch process of oil synthesis, called Gatsch. Before the beginning of the second world war the Deutsche Fettsäurewerke at Witten started production of synthetic fatty acids on an industrial scale. The acids were used by the Chemische Fabrik Imhausen \& Co. at Witten to develop production of edible synthetic fat. This made it possible to study the chemical and metabolic behaviour of synthetic fats in nutrition. Production ended in 1945 .

It was found necessary to purify the fatty acids very carefully in order to obtain from synthetic fats a white, odourless, neutral margarine without any disagreeable taste.

E. Flössner, the former chief of the Physiological Department of the Reichs Gesundheitsamt, carried through an extensive investigation of the suitability of synthetic fat for food on thousands of mice, rats, guinea-pigs, rabbits and dogs, some of the experiments extending to five or six generations (Flössner, 1948). He came to the conclusion that synthetic fat behaves like natural fat in the processes of absorption, assimilation and utilization, and that no marked changes are found in the organs of animals fed on synthetic fat. According to Flössner, a great number also of human beings consumed synthetic fat for several years without detrimental effects. In Flössner's preliminary paper, published a short time before his death, no experimental data are given of the composition of the synthetic fat or of the quantities consumed. He did not consider the fact that the consumption of synthetic fats is followed by a remarkable increase of the urinary output of dicarboxylic acids, as stated by Karl Thomas and his co-workers (Thomas \& Weitzel, 1946, 1949; Weitzel, Fretzdorff, Wojahn, Savelsberg \& Thomas, 1949). The occurrence of this aciduria shows that the metabolism is somehow altered by the use of synthetic fats.

In our laboratory at Dortmund we also have studied the digestion and assimilation of synthetic fats, but we used fats with acids having six to eight carbon atoms, not ones with long-chain fatty acids. The fatty acids which we used were not derived from Gatsch by oxidation but were normal by-products of the Fischer-Tropsch oil synthesis. Short-chain acids are essential components of butterfat, contributing from 10 to $17 \%$.

* The Food and Agriculture Organization's Nutritional Studies No. 2, published March x949, deal with synthetic fats and summarize the knowledge acquired up to 1947 (Food and Agriculture Organization, 1949). 
Glycerides of these acids form a liquid oil which, if sufficiently purified, has neither odour nor taste. It contains, like other synthetic fats, even- and odd-numbered fatty acids, of which a certain amount is of the iso-form. We found that fat from shortchain fatty acids was saponified by pancreatic lipase faster than fat from long-chain fatty acids and that, apart from small specific differences, fats from odd-numbered fatty acids were not saponified any more slowly than fats from even-numbered fatty acids (Kraut, Weischer \& Hügel, r943). When fats from short-chain fatty acids were given to rats, dogs and pigs, they were absorbed as well as other fats, and were fully metabolized, as shown by the decrease of the respiratory quotient (Kraut et al. 1944).

The growth and development of the animals were not influenced by the synthetic fat which was given daily in quantities of $2-3 \mathrm{~g}$. to rats, $10-30 \mathrm{~g}$. to dogs, and $40-135 \mathrm{~g}$. to pigs. Only those rats that had eaten less food because it contained synthetic fat showed a slower rate of increase of body-weight, but no animal had any pathological sign at autopsy (Kraut, Weischer, Hügel \& Stumpff, 1948).

It is interesting that we could not find any traces of the lower fatty acids in the body fat of these animals, in accordance with the known fact that fatty acids with chains shorter than twelve carbon atoms are immediately oxidized or synthesized to longerchain fatty acids. The presence of a small proportion of fatty acids with chains of six to twelve carbon atoms in the synthetic fats will perhaps accelerate their digestion, absorption and utilization in the body.

Recent investigations deal with the alteration of metabolism brought about by the consumption of synthetic fat. The main path of fat utilization is that of $\beta$-oxidation leading to acetic acid, but in normal fat metabolism also small amounts of dicarboxylic acids are produced which are formed by the $\omega$-oxidation of fatty acids. The occurrence of larger amounts of dicarboxylic acids in the urine after consumption of synthetic fats is not, therefore, proof of a new path of metabolism, but of a transition from the main way to a by-way of fat oxidation. It is not known whether the use of this bypath is harmful to the organism, but it should be the basis of a warning not to use synthetic fats for human nutrition before more extensive studies have been made.

Synthetic fats are distinguished from natural fats by two main characteristics: they contain about $50 \%$ of fatty acids with odd-numbered carbon chains, and they contain a large amount, from 20 to $30 \%$, of branched-chain fatty acids. In the early days of synthesis, the fat contained also a certain amount of dicarboxylic acids which were the main source of these acids excreted in the urine after consumption of synthetic fat. Unfortunately, the amount of iso-acids cannot be determined quantitatively for lack of proper methods. Thomas \& Weitzel (1949), who chiefly made the investigations, found an important diminution in the tendency to cause aciduria as the processes of the purification of synthetic fat progressed between $193^{8}$ and 1948 . To-day, dicarboxylic acids can be completely removed from the fat by careful washing with alkaline water. If, after the consumption of such washed synthetic fats, more dicarboxylic acids are found in the urine than are normally excreted this would be caused by the $\omega$ oxidation of monocarboxylic fatty acids.

The odd-numbered acids cannot be made responsible for the aciduria. We know that, like the even-numbered fatty acids, they are metabolized without the last three 
carbon atoms, which form propionic acid. By $\alpha$-oxidation propionic acid is turned into pyruvic acid, which, as well as acetic acid, enters the normal citric acid cycle of carbohydrate metabolism. Branched-chain fatty acids must, therefore, be the main cause of the increase of dicarboxylic acids in the urine. To study the behaviour of branchedchain fatty acids in the human organism is the chief physiological problem with synthetic fats.

The presence of acids in the urine may be taken as a proof of incomplete metabolism. An individual consuming $100 \mathrm{~g}$. of synthetic fat containing $20-30 \%$ of iso-acids may excrete only 3-4 g. of dicarboxylic acids in the urine, but the amount depends on the nature of the iso-acids. Appel, Böhm, Keil \& Schiller (1947) found that ethyl or propyl side chains produced far more acids in the urine than methyl side chains, amounting to from 50 to $80 \%$ of the iso-acids consumed. This is of great importance because, in the products of air oxidation of petroleum, only methyl side chains are found. Long-chain fatty acids, with the methyl group at a greater distance from the carboxyl group than the $\beta$-position are degraded by $\beta$-oxidation until the methyl group reaches the $\alpha$ - or $\beta$-position next to the carboxyl group. It depends on unknown conditions whether these shorter-chain fatty acids themselves, or dicarboxylic acids arising from them by $\omega$-oxidation, are excreted in the urine. It would seem that the existence of a large amount of such unnatural residues of $i s o$-acids is the cause of the disturbances observed such as nausea or diarrhoea.

In a recent investigation Weitzel et al. (1949) state that the position also of the methyl group in the carbon chain has great influence on the aciduria. Thus, $\beta$-methylstearic acid produces far more acids in the urine than $\alpha$ - or $\gamma$-methylstearic acid. In dodecanic acid one single methyl group attached to carbon atom 2 counteracts the oxidation of more than three methyl groups in the 3:7:I I-positions to the carboxyl. Weitzel et al. assume that this behaviour is connected with the spreading of fatty acids over the inner surfaces of the cells, which influences the action of the oxidation enzymes.

Much more investigation in this direction is certainly needed, especially of suitable methods for estimating iso-acids. We still do not know fully the reasons for, and the consequences of, the metabolic disturbances that occur after consumption of fats composed of iso-acids, but science is dealing with these problems, and it must be stated that the progress of research has led to important achievements in the industrial production of synthetic fats. Dicarboxylic acids are completely removed from the fat, non-saponifiable substances are diminished to a negligible quantity, and methods have been developed by the Badische Anilin und Sodafabrik at Ludwigshafen and the Chemische Werke Imhausen \& Co, at Witten to reduce the content of iso-acids to a very small quantity. It is, therefore, to be hoped that it will be possible to make synthetic fats fit for human consumption.

As far as I can see, there are to-day three objections that are raised to the production of synthetic fat. The first is economic. Synthetic fats cost more than natural ones. That is correct, but the facts should be remembered of the competition between natural and artificial dyes. In the early days of the chemical dye industry, artificial dyes cost much more than the same dyes of natural origin, but to-day the relationship 
is just the reverse. The second objection is that the increase in production of natural fat will bring to an end the need for synthetic fat. That may be, but the opinion of the experts, as to whether increase of fat production, or increase of the world population, is winning the race, is by no means unanimous, so it seems better to be on the safe side and produce fats in both ways. The third objection is physiological. It is claimed that what we need are not fats but fat-soluble vitamins and probably also certain essential unsaturated fatty acids neither of which are found in synthetic fats, for it is proved that the human organism can build up fat from carbohydrate or protein. In fact, to sustain life we need in our food no more fat than the small amount necessary to supply vitamins and essential fatty acids. We must, however, not only sustain life but also perform work. It was part of our experience in the war and postwar period that lack of fat in the daily diet diminished the working capacity. Heavy workers cannot take in enough calories unless a fair proportion of them is in the form of fat. Other workers need fat in their food, because consumption of fat prolongs the time of digestion and, therefore, the duration of the stream of nutrients from the intestine to the blood. This is especially important for those working the long shifts of modern industry without a rest pause for a full meal. The increase of fat consumption in all industrial countries during the last roo years is, therefore, not a matter of taste only but a necessity of modern life, so I think it a good thing to continue research on synthetic fats.

\section{REFERENCES}

Appel, H., Böhm, H., Keil, W. \& Schiller, G. (1947). Hoppe-Seyl. Z. 282, 220.

Flössner, E. (1948). Synthetische Fette, Beiträge zur Ernährungsphysiologie. Leipzig: Johann Ambrosius Barth.

Food and Agriculture Organization of the United Nations (1949). Synthetic Fats-Their Potential Contribution to World Food Requirements. Washington: Food and Agriculture Organization.

Kraut, H., Weischer, Ä. \& Hügel, R. (1943). Biochem. Z. 316, 96.

Kraut, H., Weischer, A. \& Hügel, R. (1944). Biochem. Z. 317, 187.

Kraut, H., Weischer, A., Hügel, R. \& Stumpff, G. (1948). Biochem. Z. 318, 472.

Thomas, K. \& Weitzel, G. (1946). Dtsch. med. Wschr. 71, 18.

Thomas, K. \& Weitzel, G. (1949). Klin. Wochenschr. (In the Press.)

Weitzel, G., Fretzdorff, A., Wojahn, J., Savelsberg, W. \& Thomas, K. (I949). Nature, Lond., 163, 406.

\section{The Quantitative and Qualitative Investigation of Fat Absorption}

By A. C. Frazer, J. M. French, H. G. Sammons, G. Thomas and M. D. Thompson Department of Pharmacology, University of Birmingham, and the Queen Elizabeth Hospital

Since the classical investigations of Munk \& Rosenstein ( $189 \mathrm{r}$ ), the ways of studying fat absorption in human subjects have developed singularly little. The object of this paper is to present a number of new methods which are now available for the purpose.

General study of fat absorption

\section{Total amount of fatty material absorbed}

For estimating the total amount of fat absorbed a balance technique is essential. The analysis of single samples of faeces gives no reliable information from which the 